\title{
Evaluating the Effectiveness of Nutritional Therapy in the McClelland Teaching Clinic at the University of Worcester
}

\author{
Miranda D Harris ${ }^{1 *}$ and Alison Benbow ${ }^{2 *}$ \\ ${ }^{1}$ Senior Lecturer MSc Nutritional Therapy, University of Worcester, England \\ ${ }^{2}$ Course leader MSc Nutritional Therapy, University of Worcester, England
}

\begin{abstract}
*Corresponding author: Miranda Harris, Senior Lecturer MSc Nutritional Therapy, University of Worcester, School of Allied Health and Community, St. John's Campus, Henwick Grove, Worcester, England, Email: m.harris@worc.ac.uk.

Alison Benbow, Course leader MSc Nutritional Therapy, University of Worcester, England, Email: a.benbow@worc.ac.uk.
\end{abstract}

Received Date: March 25, 2021

Published Date: April 21, 2021

\begin{abstract}
Introduction: NT is an evidence-based complementary therapy, which applies nutrition and lifestyle science to underlying pathophysiology, resulting in personalized health improvement advice. Efficacy depends on establishing a quality relationship between therapist and client. The aim of the pilot study was to measure the effectiveness of NT at the UoW teaching clinic using the Working Alliance Inventory-Short Revised (WAI-SR) and a symptom-specific outcome measure, Measure Yourself Medical Outcome Profile 2 (MYMOP2).

Methods: The interventional, before-and-after, within group pilot study used MYMOP2 to evaluate client symptoms, activity and wellbeing, and WAI-SR to evaluate therapist and client working relationship. Fifty-five clients and 22 students participated. Measures were taken at initial and follow-up consultation. Twenty completed measures were statistically analyzed using non-parametric tests.

Results: Clients presented with a range of symptoms, particularly digestive issues (27.3\%) and low energy (20\%). A statistically significant improvement was seen in Symptom 1 ( $\mathrm{z}=-3.69, \mathrm{p}<.001, \mathrm{r}=.82)$, Symptom $2(\mathrm{z}=-3.32, \mathrm{p}<.001, \mathrm{r}=.74)$, Activity $(\mathrm{z}=-2.07, \mathrm{p}<.001$, $\mathrm{r}=.7)$, WAI-SRT ( $\mathrm{z}=3.18$, $\mathrm{p}<.05, \mathrm{r}=.7)$ and WAI-SRC $(\mathrm{z}=2.19, \mathrm{p}<.001, \mathrm{r}=.5)$ with large effect sizes, and no significant improvement in Wellbeing. Strong, positive relationships were found between combined Symptom 1 and Activity, and Wellbeing at initial $(r=.5, n=20, p<.05)$ and follow-up ( $r=.6, n=20, p<.05)$. A strong therapist and client working alliance was evident at initial and follow-up consultation.

Conclusion: Preliminary results suggest NT practice is effective in reducing client symptoms and improving health and activity. This may be facilitated by a strong therapist and client working alliance. Further larger-scaled research would support findings with more robust analysis.
\end{abstract}

Keywords: Nutritional therapy; Working-relationship; Client; Therapist; MYMOP2; WAI-SR

Abbreviations: A: Activity; MYMOP: Measure Yourself Medical Outcome Profile; NT: Nutritional Therapy; S1: Symptom 1; S2: Symptom 2; WAI-SR: Working Alliance Inventory- Short Revised; WAI-SRC: Working Alliance Inventory-Short Revised Client; WAI-SRT: Working Alliance Inventory- Short Revised Therapist; WB: Well-being

\section{Introduction}

There is little published research on the efficacy of Complementary and Alternative Medicine (CAM) including Nutritional Therapy (NT), as revealed by a search of the Cochrane Database of Systematic reviews (accessed 5th December 2019). This was highlighted at the Research Council for Complementary Medicine
(RCCM) conference "Working with others and Collaboration in research" in 2019, where the use of the evaluation tool, Patient Reported Outcome Measure (PROMS) was discussed as an area for future attention (personal communication), to enhance the evidence base for CAM professions. NT is an evidence-based complementary 
therapy, which applies nutrition and lifestyle science to underlying pathophysiology, resulting in personalised recommendations for health improvement [1]. The development of the profession, including educational provision, has been ongoing since the 1980's [2] and has resulted in practice standards [3,4] and accredited qualifications [5], which aim to generate safe, evidenced, effective and legal practitioners. NT efficacy may depend on establishing a quality relationship between the therapist and client based on mutual respect, communication and understanding of roles and responsibilities [6]. A recent evidenced-based update [7] of the Core Curriculum for NT [8], suggested the profession may benefit further from improved educational provision to develop the therapist-client relationship, which includes behaviour change theory and strategy. The establishment of appropriate communication skills to develop successful, therapeutic relationships for effective clinical practice, including the application of behaviour change techniques endeavors to support, motivate, and empower clients to make health changes, as outlined in the National Occupational Standards for NT (CNHC 1,2,8 and 9) [9-12]. Further exploration of this therapeutic relationship, to encompass the establishment of health goals and the prerequisite actions, as well as the readiness to change behavior may result in improved client outcomes and contribute to the learning and teaching of clinical skills for the NT training providers. A study by Harris, et al. [13] evaluated the relationship between therapist and client, while examining the effectiveness of aromatherapy, massage and reflexology in clinical practice in a student clinic; they concluded that the symptoms, activity and wellbeing were associated with a positive working therapeutic alliance, but this did not achieve statistically significant. As there were no similar studies in NT it was decided to develop this study specifically in NT using the same instruments as Harris, et al. [13], namely the Measure Yourself Medical Outcome Profile 2 (MYMOP2) and the Working Alliance Inventory-Short Revised (WAI-SR).

\section{Measure Yourself Medical Outcome Profile (MY- MOP)}

MYMOP is a patient/client centred outcome measure, which was originally designed by primary care practitioners $[14,15]$ and since used in complementary health care including NT. This objective measure, which is quick and easy to complete in a consultation, enables the client to focus on aspects of their health and identify factors for change, thus facilitating a more clientfocused relationship $[13,16,17]$. The client identifies two specific health complaints or symptoms, which have been present over the past week, one activity limited by the symptoms and a general wellbeing score. The client repeats this process at follow-up using MYMOP Follow Up (FU), without sight of the previous scores of MYMOP. MYMOP profile scores are calculated by averaging the four item scores, which can be compared over subsequent consultations with the items measured remaining the same [18]. A second iteration was introduced in 2000, MYMOP2 [19], which includes a medication question and an option to express the importance of reducing or ceasing this medication [17]. This question is not measured and therefore does not contribute to the final MYMOP2 score.

\section{Comparison with other measures}

MYMOP/MYMOP2 has been evaluated extensively and proved to be reliable [20], valid and responsive [18], in particular for the symptoms and activity questions [14]. When compared to other measures such as health status questionnaire SF-36 [14], EuroQol5D (EQ5D), COOP WONCA charts [21], and Medical Outcomes Study 6-item general health survey (MOS-6A) [19], MYMOP/MYMOP2 seems conceptually aligned to a therapy, which is personalized and measures benefits experienced by the client, not necessarily captured by traditional instruments [22].

\section{Use of MYMOP}

There are several examples of the use of MYMOP/MYMOP2 to evaluate CAM therapy, including a study on the use of acupuncture for pain management, which demonstrated clinically significant health improvements [17]; the addition of Healing Therapy with conventional treatment in Irritable Bowel Syndrome (IBS) and Inflammatory Bowel Disease (IBD), which was associated with improvement in symptoms [23] and a demonstration of efficacy of the Alexander Technique in improving back pain [21]. Ishaque, et al. [22] executed a critical review of 16 studies using MYMOP and adaptions of MYMOP in therapies such as cancer, psychiatry and acupuncture, and suggested that MYMOP does offer individualized assessment of patient-centred outcomes and contributes to the understanding of the "heterogeneity of treatment effects" and although further validation maybe beneficial, it was considered suitable for this study.

\section{The Therapeutic Relationship}

The relationship between the therapist and client/patient, known as the therapeutic relationship, alliance or bond [24,25], has repeatedly shown to be an effective outcome predictor for success [26]. The original conceptualization of the impact of the "therapeutic relationship" on treatment success [27,28] was adapted by Horvath \& Greenberg [29]. There is no single definition of the term, but the core tenets suggest the alliance is a helping, collaborative relationship which is dynamic, may involve mutual trust, confidence and commitment, with goals that relate to a shared sense of purpose. Recognition of the collaborative bond between healer and patient dates back to the writings of Hippocrates [24,30], and more recently to Freud in the early 20th century [24], where the concept was explored extensively [30]. The pivotal point of this perspective was transference, and how the relationship between healer and patient reflects varying aspects of this process, a perspective supported by other therapists such as Klein [30]. On the other hand, Freud recognized the validity of the therapist's attitude to the client and other conscious aspects such as compassion and kindness, a non-transferential process 
corroborated by psychoanalysts Zetzel [25] and Greenson [31,32]. Although the history of the alliance through the 20th century is a fascinating study, it goes beyond the scope of this article, and it is suffice to report these divergent views were fused and common factors identified to form a framework for the working alliance, a pantheoretical construct to include goals, tasks and affective bonds as described by Bordin [33,34]. The alliance became an effective tool for researching the role of the relationship in a wide range of therapies, including psychotherapy [30].

\section{The Working Alliance Inventory (WAI) and Devel- opment}

The Working Alliance Inventory (WAI) is a validated measure used to assess the quality of therapeutic relationships between therapist and client using two questionnaires WAI-Therapist (WAI-T) and WAI-Client (WAI-C), which is considered to predict successful therapeutic outcomes [24]. It assesses three key elements; agreement between therapist and client on the goals of therapy, consensus on the tasks of therapy to address the issues identified by the client, and the development of an effective bond between the two parties $[24,33,34]$. Different scales have been designed in order to measure the three domains of the alliance, including the Session Rating Scale (SRS) Version 3 [26], the Helping Alliance Questionnaire [35] and the Working Alliance Inventory-36 item [36], a theory-based approach developed by Horvath \& Greenberg [20] and the WAI-Short-12 item developed by Tracey \& Kokotovic [37], a shorter more versatile version, suitable for repeated measurements [38]. A further iteration, the WAI-SR 12 item was validated in 2006 and results demonstrated effective differentiation of the dimensions of goals, task and bonds [33], and due to removal of negative words, confirmatory factor analysis (CFA) showed an improved model fit [39].

\section{Use of the WAI}

Since its inception it has been widely used and reviews have shown it is a robust predictor of therapy outcome [40]. Use has not been confined to psychotherapy but has been broadened to include both medical and physical rehabilitation, including a 12week cardiac rehab programme to measure overall physical and mental health, cardiac fitness and perceived exertion [41]. In other areas exploration of its validity has included the child protection services, which supported its use in practice and research, showing convergent construct validity with other measures [42]. Jasper, et al. [34] compared the outcome of group CBT and internet CBT on tinnitus, and reported a strong working alliance between both groups, with moderate relationships between the alliance and outcome. In primary care obesity interventions, where the WAI was used between GP's and their patients, the therapeutic alliance was strongly correlated with patient engagement; this study reported the strength of the alliance was associated with the ability to carry out usual tasks, as well as emotional wellbeing and self-esteem, and results confirmed effective measurement of the GP-patient alliance [43].

\section{Aim and Objectives of the Study}

There is little literature around the efficacy of NT as a CAM therapy, so as well as exploring therapeutic relationships and their contribution to effectiveness, this study aimed to contribute to the continuing professional development including educational provision, and if successful could be used as a model for establishing effective practice in the wider NT arena as well as in the teaching environment.

The aim was to measure and evaluate the effectiveness of NT in a teaching clinic using WAI-SRT, WAI-SRC and MYMOP2, and the objectives were to:

- Investigate the relationship between the student therapist and the client using WAI-SRT and WAI-SRC.

- $\quad$ Evaluate the effectiveness of NT comparing MYMOP2 and MYMOP2FU.

- $\quad$ Predict the potential outcome of NT using WAI-SRT and WAI-SRC, and MYMOP2.

- $\quad$ Formulate future strategies for the development of therapeutic relationships in a NT teaching and learning environment.

\section{Methods}

An interventional, before and after, within group pilot study was carried out in the McClelland clinic at the University of Worcester (UoW), where NT MSc student's complete client consultations during their clinical practice module in order to achieve the required Skills for Health, National Occupational Standards (NOS). Although there are different methods of data collection to evaluate when considering a research strategy, including quantitative, qualitative, and mixed methods, since this research was collecting facts, and studying the relationship between them, a quantitative method was selected $[44,45]$.

\section{Participants}

Students were recruited from the McClelland clinic and clients were recruited from the UoW and the general public. All participants were 18 or over and recruited between October 2017 and July 2018. There were no exclusion criteria unless a client had been considered unsuitable for NT during the first consultation. As the sample size may affect power, a calculation of the number of participants needed to detect a statistically significance in tests of differences and relationships was computed as 60 [46], however due to time restrictions this was subject to student and client availability and willingness to take part in the study.

\section{Ethical approval}

Before students and clients were invited to take part in the research, Ethical Approval was granted by the Health \& Science Ethics Research Committee at the UoW HSREC code SH17180022. Participant information was supplied for consideration before 
the participants were asked to give written consent. Participation was voluntary for both clients and students, and there were no incentives offered for taking part. Students could decide not to take part without any risk or penalty to the clinic assessment process. Clients and students were able to withdraw from the study up until 30 September 2018. No potential risk of harm was identified. The clinic operates to the CNHC and BANT Professional Practice Codes, which include client confidentiality and storage of records $[3,4,47]$. Clients and therapists were assigned a study number for anonymity, which was used on all documentation. Both researchers were CNHC registered nutritional therapists and members of the professional body BANT.

\section{Data collection}

Pre-validated instruments MYMOP2 and MYMOP2FU, WAISRC and WAI-SRT (Appendix A, B, C \& D) were chosen as the most suitable and effective instruments for efficient fact finding in this study [44, 48]. Minor adjustments were made to the wording of the WAI-SRT and WAI-SRC to make it specific to NT by replacing the word therapy/therapist with Nutritional therapy/Nutritional therapist, respectively. The client demographic data was gathered from the anonymized clinical records. Data was collected during the consultation period in the McClelland teaching clinic where students were supervised and assessed. Students saw a minimum of three clients and each client has three consultations; Initial 1 to take the case history, Initial 2 to deliver NT recommendations and the third to follow up on compliance and make further recommendations. Each appointment was approximately one hour with a minimum of three weeks in between each session. MYMOP2 and MYMOP2 FU data were collected from the client during the consultations, and WAI-SRT and WAI-SRC data were completed by therapist and client respectively at the end of the consultations. The rest of the consultation proceeded as normal. Clients rated symptom 1 (S1), symptom 2 (S2) and wellbeing (WB) using a 7-point Likert scale on MYMOP2 and MYMOP2FU with the range of ( 0 , as good as it could be to 6 , as bad as it could be). Clients also rated an activity (A), which was affected by one of the symptoms using the same scale. Clients and therapists rated the statements on the WAI-SR questionnaires using a 5-point Likert scale 1-5. Clients rated 12 statements and students rated 10 statements.

\section{Data Analysis}

MYMOP 2 scores from the four items of S1, S2, WB and A were summed at I1 and FU and mean scores were compared using tests of differences and relationships. Scores of S1 and S2, and S1 and A were combined for further tests. WAI-SRC and WAI-SRT scores were summed following I1 and FU and mean WAI-SR scores were calculated for client and therapist for quantitative tests of differences and relationships. Data was entered into SPSS 23 and assessed for normal data distribution and assumptions for each set of tests. Due to the results, non-parametric tests were chosen: the Wilcoxon Signed Rank Test for repeated measures and Spearman's correlation coefficient test for relationships. Only data from completed sets of MYMOP2, WAI-SRT and WAI-SRC were analyzed using SPSS $23(\mathrm{n}=20)$, however descriptive analysis was carried out on all data to report on the characteristics of clients visiting the clinic during the study.

\section{Results}

The total number of clients attending the clinic was 74, with 55 taking part in the research. Out of 24 students in the 2017 /8 cohort, 22 took part. The total number of study consultations was 169 with an average of 3.07 ( $S D=0.38$ ) consultations per client. Fifty clients received three consultations; of the rest, one had two, three had four and one had five sessions. The mean client age was 44.2 years $(n=55)$ (SD 11.83) with a range of 19-72. The most common age was 54 (12.73\%). Of the 55 clients, 48 were female and 7 were male. The clients presented with a range of symptoms see Table 1 as determined by MYMOP2; the most common reported for S1 $(\mathrm{n}=55)$ and S2 (n=52) was digestion (S1 25.45\%, S2 23.07\%) and energy (S1 21.81\%, S2 15.38\%). The length of time reported for S1 $(n=40)$ ranged from 4-12 weeks to over five years with the greatest number reporting symptoms for over 5 years (42.5\%) (Figure 1 \& Table 1).

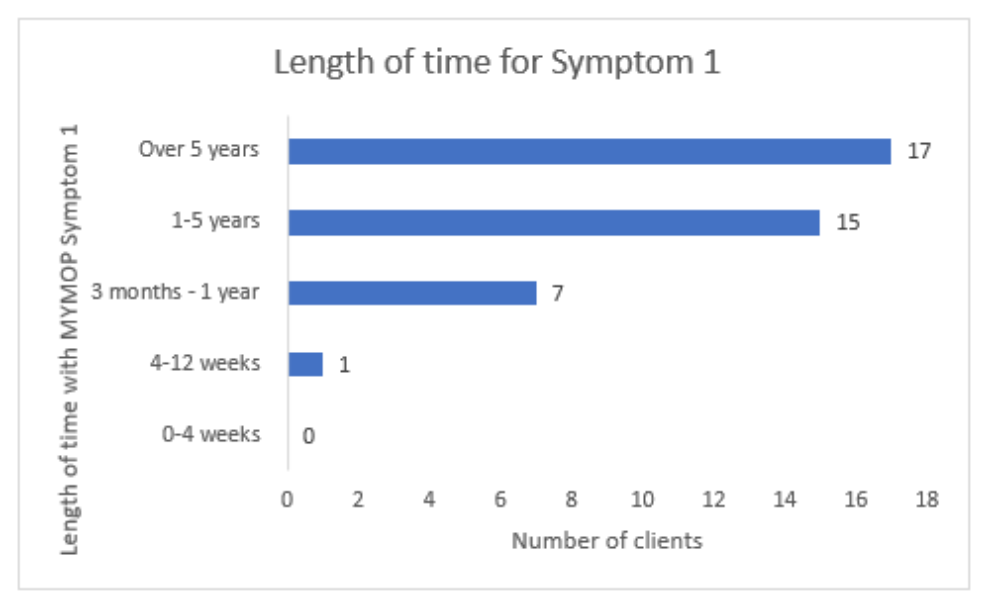

Figure 1: Length of time with MYMOP 2 symptom 1. 
Table 1: MYMOP2 Symptoms 1 and 2 at Initial 1.

\begin{tabular}{|c|c|c|c|c|}
\hline Symptom & Symptom 1 (S1) & \% & Symptom 2 (S2) & 23.07 \\
\hline Digestion & 14 & 25.45 & 8 & 12.38 \\
\hline Energy & 12 & 21.81 & 3 & 5.77 \\
\hline Weight & 8 & 14.55 & 4 & 7.69 \\
\hline Exercise performance & 5 & 9.09 & 2 & 3.85 \\
\hline Respiratory & 3 & 5.45 & 5 & 1.92 \\
\hline Joint pain & 3 & 5.45 & 3 & 9.62 \\
\hline Nutrition knowledge & 2 & 3.64 & 3 & 5.77 \\
\hline Female hormones & 2 & 3.64 & 1 & 5.77 \\
\hline Stress & 2 & 3.64 & 5 & 1.92 \\
\hline Skin issues & 2 & 3.64 & 2 & 9.62 \\
\hline Mood & 1 & 1.82 & 2 & 3.85 \\
\hline Sleep & 1 & 1.82 & 1 & 3.85 \\
\hline Headaches & 0 & 0 & 52 & 1.92 \\
\hline Skin issues & 0 & 0 & & \\
\hline Total & 55 & & & \\
\hline
\end{tabular}

Activities represented by MYMOP2 $(\mathrm{n}=43)$ ranged from social activities (30.23\%), general activity performance $(27.90 \%)$ and exercise performance (25.58\%) see Figure 2. The wellbeing scores $(n=38)$ showed an improvement from an average score of 2.47 to 1.93 . Of the clients who responded to questions about taking medication for $\mathrm{S} 1(\mathrm{n}=50), 38$ were not taking medication and of the 12 taking medication, 6 stated it was 'very important' to reduce and 3 suggested it was 'a little important' and one said it was 'not important' (Figure 2).

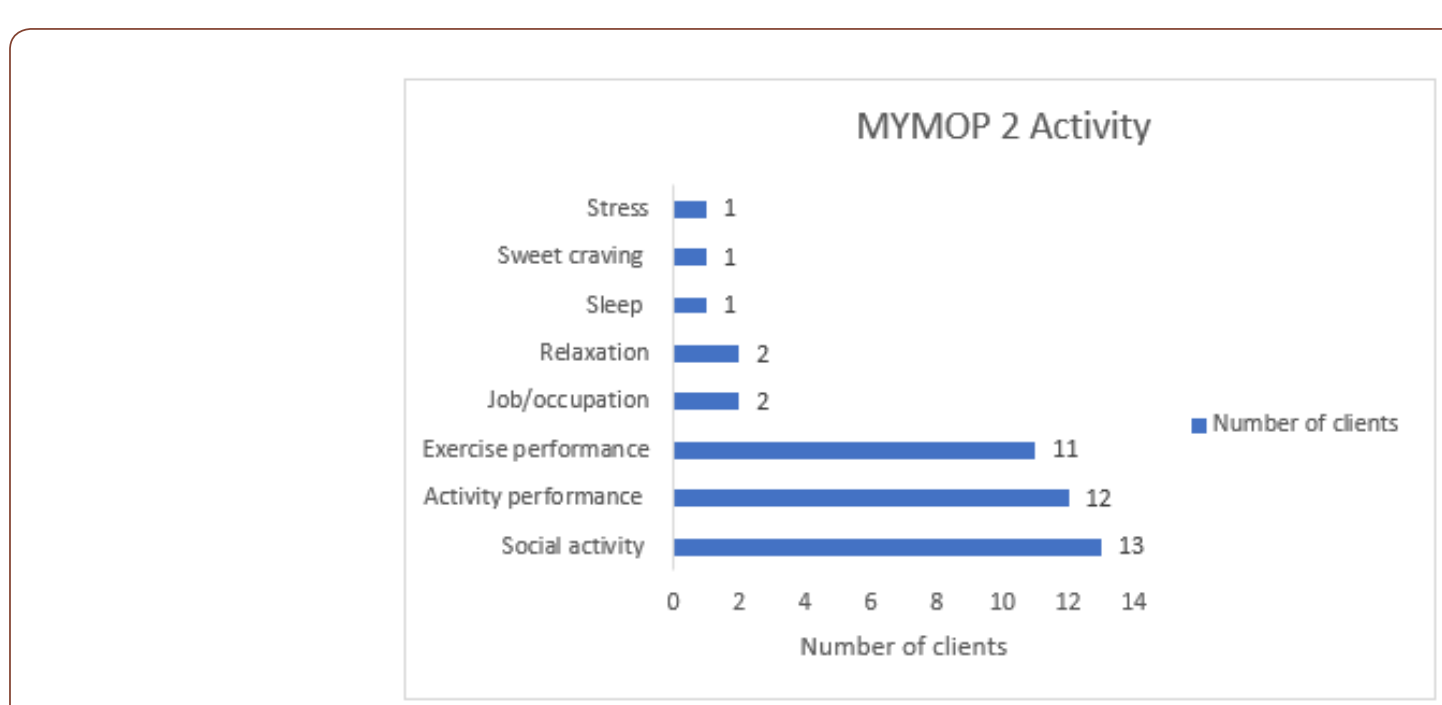

Figure 2: MYMOP 2 Activity.

The relationship between the student therapist and the client using WAI-SRT and WAI-SRC

Mean scores for the WAI-SRT and WAI-SRC were calculated

Table 2: WAI-SRT and WAI-SRC at I1 and FU.

\begin{tabular}{|c|c|c|}
\hline WAI-SR & I1 mean (SD) & FU (mean SD) \\
\hline WAI-SRT & $43.3(4.99)$ & $45.6(3.58)$ \\
\hline WAI-SRC & $51.8(6.2)$ & $57.05(3.46)$ \\
\hline
\end{tabular}

at $\mathrm{I} 1$ and FU, to measure the strength of the relationship of the working alliance. Scores demonstrated a strong working alliance for therapist ( 43.3 and 45.6 out of a total 50 ) and client (51.8 and 57.05 out of a total 60 ) at I1 and FU respectively (Table 2). 
Table 3: MYMOP2 scores at 11 and FU.

\begin{tabular}{|c|c|c|}
\hline MYMOP2 & I1 [mean (SD)] & FU [mean (SD)] \\
\hline S1 & $4.4(0.75)$ & $2.3(1.45)$ \\
\hline S2 & $3.85(1.04)$ & $2.1(1.25)$ \\
\hline S1S2 combined & $8.25(1.33)$ & $4.4(2.19)$ \\
\hline A & $4.2(2.41)$ & $2.5(1.5)$ \\
\hline W & $2.47(1.12)$ & $1.93(1.16)$ \\
\hline S1A combined & $7.55(2.42)$ & $3.45(2.35)$ \\
\hline
\end{tabular}

A statistically significant improvement was seen between I1 and FU in WAI-SRT $(\mathrm{z}=3.18, \mathrm{p}<.05, \mathrm{r}=.7)$ and WAI-SRC $(\mathrm{z}=2.19$, $\mathrm{p}<.001, \mathrm{r}=.5$ ) with large effect sizes [49].

\section{The effectiveness of NT comparing MYMOP2 and MYMOP2FU}

Mean scores of S1, S2, A and W were calculated at I1 and FU see Table 3. A statistically significant improvement was seen between $\mathrm{I} 1$ and FU in S1 ( $\mathrm{z}=-3.69, \mathrm{p}<.001, \mathrm{r}=.82), \mathrm{S} 2 \quad(\mathrm{z}=-3.32, \mathrm{p}<.001$, $\mathrm{r}=.74), \mathrm{A}(\mathrm{z}=-2.07, \mathrm{p}<.001, \mathrm{r}=.7)$, all with large effect sizes, and no statistically significant improvement was seen in WB $(z=-1.45$, $\mathrm{p}=.147, \mathrm{r}=.1$ ) (Table 3).

The potential outcome of NT using WAI-T and WAI-C, and MYMOP2

Mean scores of S1S2 combined and S1A combined were calculated at I1 and FU see Table 3. Correlations looked for relationships in mean scores of WAI-SRC and WAI-SRT and differences in S12 scores between I1 and FU. A medium, positive relationship was found between WAI-SRT I1 and differences in S12 scores between I1 and FU ( $\mathrm{r}=.38, \mathrm{n}=20, \mathrm{p}=.10)$ and a small, positive relationship at FU ( $\mathrm{r}=.20, \mathrm{n}=20, \mathrm{p}=.39)$. Small, positive relationships were found between WAI-SRC (I1 and FU) and differences in S12 scores between I1 and FU (r=.10, n=20, p= .83; r=.15, n=20, p=.52) respectively. These results suggest a positive working alliance is associated with improved symptoms, however, none of these relationships reached statistical significance. Correlations looked at mean scores of combined S1 and $\mathrm{A}$, and WB to find strong, positive relationships [49] at I1 $(r=.5, n=20, p<.05)$ and $F U(r=.6$, $\mathrm{n}=20, \mathrm{p}<.05$ ) achieving statistical significance, suggesting wellbeing scores vary with symptom and activity level.

\section{Discussion}

Data collected from 55 clients reported the average client was female, mid-fifties and had attended three consultations. According to MYMOP2 data approximately $25 \%$ of clients reported digestive symptoms for symptom 1 and $23.07 \%$ reported digestive symptoms for symptom 2. A recent review article divulged that a significant proportion of patients suffering from function gastrointestinal issues turn to complementary and alternative therapies with satisfactory outcomes [50]. The second most common symptom for both symptom 1 and 2 was energy, and other presenting symptoms included weight loss, mood and 'exercise performance'. It is difficult to compare these results with other NT practice, however there is a plethora of evidence to support personalized nutritional strategies for weight loss [51,52], increasing energy, and enhancing sports and exercise performance [53]. Of the clients who responded, $42.5 \%$ had symptom 1 for more than five years. Activities affected by symptoms included social activity, and exercise and activity performance. Of those taking medication $(n=12), 50 \%$ stated it was very important to stop, however this was less than a quarter of those who responded to the question so may not be representative of those attending clinic. A qualitative study which evaluated 327 patients attending an NHS outpatient department offering acupuncture, osteopathy, and homoeopathy, highlighted key reasons to seek complementary therapy included concerns about taking medication, particularly on a long-term basis [54].

\section{Relationship between the student therapist and the client using WAI-SRT and WAI-SRC}

High mean scores were reported from both student therapist and client at I1 and FU consultations (see Table 2) thus suggesting a strong working relationship, which has been evident in other research $[13,36]$. To the author's knowledge, the WAI has not been used in NT before, however a recent study using an adapted form of the WAI, known as the Working Alliance Inventory Observerrated Short Version (WAI-O-S) found it effective in measuring the therapeutic alliance in dietetic consultations for weight loss [55]. Interesting patients who completed food diaries prior to attending a health coaching session for weight loss, as was carried out in this research, were able to engage well with the dietitian and develop a stronger therapeutic alliance [56]. In addition, the therapeutic alliance has been positively correlated to favorable outcomes in chronic disease and weight loss [41].

\section{Effectiveness of NT comparing MYMOP2 and MYMOP2FU}

Statistically significant improvements were seen between I1 and FU in S1, S2 and A with a large effect size reported (see Table 3 ) thus implying the effectiveness of NT, although confounding factors may have influenced the results, such as client expectations [13], with those who agreed to take part in the research having more positive expectations $[17,18]$. There was no statistical significance improvement in WB between 1 and FU, however there was indication of a tendency for WB to vary with S1 and A scores as seen in Harris [13]. The reported results for improvement in symptoms, and activity affected by symptoms is encouraging and further larger-scale research is recommended in light of the small sample size in this research [56]. As seen in other research, MYMOP2 is a useful individualized outcome assessment tool, which has been used extensively in complementary therapies [22]. As a client-led instrument, it allows the client to identify aspects of health and lifestyle of most concern and given the current move to a more personalized therapeutic approach, MYMOP and its various iterations appears to offer an excellent fit to this more individualized approach [22]. Compared to other more traditional 
'quality of life' and 'health outcome' measures with predetermined domains, which may not represent health domains valued by the client [22], this tool allows the client to monitor progress in a clinical scenario and may serve as a useful learning aid in teaching clinics $[20,57]$, where the student can evaluate the effectiveness of their recommendations.

\section{The potential outcome of NT using WAI-SRT and WAI- SRC, and MYMOP2}

Results suggest the WAI-SR may be helpful in determining the predictability of the working alliance on outcome of NT. The relationship between WAI-SRT I1 and difference in symptom scores between I1 and FU indicated the stronger the working alliance of the therapist initially, the more MYMOP2 symptom improvement was seen between I1 and FU. The relationship between WAI-SRC I1 and difference in MYMOP2 symptom scores between I1 and FU suggested the strength of the client working alliance may also impact on their symptom improvement. However, as neither of these relationships reached significance, further research with a larger sample size would be recommended before robust conclusions can be drawn on the impact of the working alliance on NT outcomes. Although this study did not look at the psychological construct of the working alliance, other recommendations for further research could include individual assessment of the three dimensions which characterize the alliance, namely goals, tasks, and bonds [58]. This may be helpful to determine the impact of factors such as shared goal setting and decision-making on the outcome of therapy [43]. It is worth mentioning some research suggests the two dimensions of goals and tasks could be construed as one factor due to the high correlations between these two dimensions $[59,60]$. Strong, positive relationships, which reached significance were found between combined S1 and A, and WB at I and FU as seen in previous research [13], with high levels of Symptom 1 and activity improvement scores being associated with higher levels of wellbeing improvement scores. These results support the use of MYMOP2 in clinical teaching and practice as an effective measure of NT and therefore can be regarded as a positive development to the profession.

\section{Formulate future strategies for the developments of therapeutic relationships in a NT teaching and learning environment}

As the therapeutic alliance has been positively reported on in this study, as well as extensively in other research including conditions of chronic disease, it is recommended that the WAISRT/C is included in clinic assessment using online measures for ease and compliance (NTEC 2017). To further address the issue of non-compliance to the paperwork, in particular WAI-SRT/C as seen in this study, consideration should be given to other measures such as the ultra-brief alliance measure SRSV3, which has been designed for use by clinicians rather than for research purposes and has strong internal consistency for the overall measure, as well as four subscales [24]. Both working alliance and MYMOP measures are strongly recommended for use in clinical practice for all NT's, who would benefit from the predictability of the measures as well as the measurement of effectiveness practice.

\section{Limitations and suggestions for further research}

Completion of the paperwork was very poor which affected the sample size for analysis, and consequently power resulting in the chance of Type 2 errors, where a difference may not have been detected when there was one, therefore a larger sample size for more robust statistical analysis would be recommended for future research [61]. In addition, it may be beneficial to compare MYMOP effectiveness and the working alliance relationship in gender and age categories, as well as morbidity characteristics [62]. Further research may help identify other skills necessary to build on the therapeutic alliance for more effective health behavior change [41].

\section{Conclusion}

In conclusion, despite limitations this pilot study has provided preliminary results, which demonstrate through the use of two validated measures the effectiveness of NT as an evidence-based therapy for health and lifestyle improvements and their use in predicting NT efficacy by the strength of the working alliance between therapist and client. Not only do the results contribute to the development of the profession but may be transferable to other areas in CAM.

\section{Acknowledgement}

The authors would like to thank the students and clients who took part in this study and the administrative staff at the McClelland Centre for their contribution.

\section{Conflict of interest}

None.

\section{References}

1. British Association of Applied Nutrition and Nutritional Therapy (2019) About Nutritional Therapy.

2. British Association for Applied Nutrition and Nutritional Therapy (2020) Brief history of BANT and Nutritional Therapy.

3. British Association of Applied Nutrition and Nutritional Therapy (2020) Professional Practice Handbook.

4. Complementary and Natural Health Care Council (2018) Code of Conduct for Registrants.

5. Nutritional Therapy Education Commission (2020) Are you considering training as a Nutritional Therapist.

6. Fejzic J, Emmerton L, Tett SE (2010) Towards concordance in healthcare: perspectives of general practitioners, complementary and alternative medicine practitioners and pharmacists in Australia. J Clin Pharm and Ther 35(3): 309-321.

7. Benbow A, Ralph S, Watkins K, Granger C (2017) Exploring the Current Working Profiles of Nutritional Therapists to Inform Curriculum and Professional Development. Eur J Integr Med 23-31.

8. Nutritional Therapy Education Commission (2017) Core Curriculum for Nutritional Therapy. 
9. Skills for Health (2010) Explore and establish the client's needs for complementary and natural healthcare.

10. Skills for Health (2010) Develop and agree plans for complementary and natural healthcare with clients.

11. Skills for Health (2010) Provide nutritional therapy to clients.

12. Skills for Health (2010) Prescribe nutraceuticals to.

13. Harris P, Atkins RC, Alwyn T (2010) Evaluating a complementary therapies clinic: Outcomes and relationships. Complement Ther Clin Pract 16(1): 31-35

14. Paterson C (1996) Measuring outcome in primary care: a patientgenerated measure, MYMOP, compared to the SF-36 health survey. Br Med J 312(7037): 1016-1020.

15. Paterson C, Thomas K, Manasse A, Cooke H, Peace G (2007) Measure Yourself Concerns and Wellbeing (MYCaW): An individualised questionnaire for evaluating outcome in cancer support care that includes complementary therapies. Complement Ther Med 15(1): 3845 .

16. Paterson C (2006) Measuring changes in self-concept: a qualitative evaluation of outcome questionnaires in people having acupuncture for their chronic health problems. BMC Complement Alt Med 16: 6-7.

17. Vernooij M, Marcelissen F (2017) Measuring patient reported outcomes of acupuncture treatment on pain patients' health status. Complement Ther Clin Pract 28: 192-199.

18. Kristoffersen AE, Stub T, Knudsen-Baas O, Udal A, Musial F (2019) Selfreported effects of energy healing: a prospective observational study with prepost design. Explore 15(2): 115-125.

19. Paterson C, Britten N (2000) In pursuit of patient-centred outcomes: a qualitative evaluation of MYMOP, Measure Yourself Medical Outcome Profile. J Health Serv Res Policy 5(1): 27-36.

20. McClean S, Brilleman S, Wye L (2015) What is the perceived impact of Alexander technique lessons on health status, costs and pain management in the real life setting of an English hospital? The results of a mixed methods evaluation of an Alexander technique service for those with chronic back pain. BMC health services res 15: 293.

21. Paterson C (2004) Seeking the patient's perspective: A qualitative assessment of EuroQol, COOP-WONCA charts and MYMOP. Qual Life Res 13(5): 871-881.

22. Ishaque S, Johnson JA, Vohra S (2019) Individualized health-related quality of life instrument Measure Yourself Medical Outcome Profile (MYMOP) and its adaptations: a critical appraisal. Qual Life Res 28(4): 879-893.

23. Lee RT, Kingstone T, Roberts L, Edwards S, Soundy A (2017) A pragmatic randomised controlled trial of healing therapy in a gastroenterology outpatient setting. Eur J Integr Med 9: 110-119.

24. Martin DJ, Garske JP, Davis MK (2000) Relation of the therapeutic alliance with outcome and other variables: a meta analysis review. J Consulting Clin Psychol 68(3): 438-450.

25. Zetzel ER (1956) Current concepts of transference. Int J of Psychoanal 37(4-5): 369-376.

26. Duncan BL, Miller SD, Sparks JA, Claud DA, Reynolds LR, Brown J, Johnson LD (2003) The Session Rating Scale: Preliminary Psychometric Properties of a Working Alliance Measure. J Br Ther 3(1).

27. Bordin ES (1979) The generalizability of the psychoanalytic concept of the working alliance. Psychotherapy: Theory Research \& Practice 16(3): 252-260.

28. Bordin ES (1994) Theory and research on the therapeutic working alliance: New directions, In: Horvath AO, Greenberg LS (Edn.), The working alliance: Theory research and practice Wiley New York, pp.281304.

29. Horvath AO, Greenberg LS (1989) Development and Validation of the Working Alliance Inventory. J Counc Psychol 36(2): 223-233.
30. Horvath AO (2018) Research on the alliance: Knowledge in search of a theory. Psychother Res 28(4): 499-516.

31. Greenson RR (1965) The working alliance and the transference neurosis. The Psychoanal Q.77(1): 77-102.

32. Greenson RR (1990) The working alliance and the transference neurosis. In: Esman AH, Esman AH (Eds.), Essential papers on transference, New York University Press, New York, pp. 150-171.

33. Hatcher RL, Gillaspy JA (2006) Development and validation of a revised short version of the working alliance inventory. Psychother Res 16(1): $12-25$.

34. Jasper K, Weise C, Conrad I, Andersson G, Hiller W, et al. (2014) The working alliance in a randomized controlled trial comparing Internetbased self-help and face-to-face cognitive behavior therapy for chronic tinnitus. Internet Interv 1(2): 49-57.

35. Luborsky L, Barber J, Siqueland L, Johnson S, Najavits L, et al. (1996) The Revised Helping Alliance questionnaire (HAQ-II): Psychometric properties. The J Psychother Pract Res 5(3): 260-271.

36. Paap D, Dijkstra PU (2017) Working Alliance Inventory-Short Form Revised to measure therapeutic alliance. J Physiother 63: 2.

37. Tracey TJ, Kokotovic AM (1989) Factor structure of the Working Alliance Inventory. Psychol Assess 1:207-210.

38. Busseri MA, Tyler JD (2003) Interchangeability of the Working Alliance Inventory and Working Alliance Inventory Short Form. Psychol Assess 15(2): 193-197.

39. Munder T, Wilmers FR, Leonhart R, Linster HW, Barth J (2010) Working Alliance Inventory-Short Revised (WAI-SR): psychometric properties in outpatients and inpatients. Clin Psychol Psychother 17(3): 231-239.

40. Horvath AO, Del Re AC, Flückiger C, Symonds D, Alliance Indiv (2011) Psychother. 48: 9-16.

41. Burns JW, Evon D (2007) Common and Specific Process Factors in Cardiac Rehabilitation: Independent and Interactive Effects of the Working Alliance and Self-Efficacy. Health Psychology Am Psychol Assoc 26(6): 684-692.

42. Killian M, Forrester D, Westlake D, Antonopoulou P (2017) Validity of the Working Alliance Inventory Within Child Protection Services. Res. Social Work Pract 27(6).

43. Sturgiss EA, Rieger E, Matthew EH, Ridd J, Douglas K, et al. (2019) Adaption and validation of the Working Alliance Inventory for General Practice: qualitative review and cross-sectional surveys. Family Pract 36(4): 516-522.

44. Bell J, Waters S (2018) Doing Your Research Project: A Guide for First Time Researchers. ( $7^{\text {th }}$ edn.), McGraw-Hill Education, London.

45. Green J, Thorogood N (2014) Qualitative methods for health research ( $3^{\text {rd }}$ edn.), Sage Publications Ltd, London.

46. Wilson Van Voorhis CRW, Morgan BL (2007) Understanding power and rules of thumb for determining sample sizes. Tutorials in Quantitative Methods for Psychology 3(2): 43-50.

47. University of Worcester (2018) Data Protection Policy.

48. Denscombe M (2014) The Good Research Guide for small-scale social research projects. $\left(5^{\text {th }}\right.$ edn.), [e-book] Open University Press, Maidenhead, England.

49. Cohen J, Power Primer A (1992) Quant Methods Psychol. 112(1): 155159.

50. Jill Deutsch K MD, Levitt J, Hass DJ (2020) Complementary and Alternative Medicine for Functional Gastrointestinal Disorders. Am J Gastroenterol 115(3): 350-364.

51. Freire R (2020) Scientific evidence of diets for weight loss: Different macronutrient composition, intermittent fasting, and popular diets. Nutrition. 69: 110549 
52. Lombardo M, Perrone MA, Guseva E, Aulisa G, Padua E, et al. (2020) Losing Weight after Menopause with Minimal Aerobic Training and Mediterranean Diet. Nutrients 12: 2471

53. Beck KL, Thomson JS, Swift RJ, von Hurst PR (2015) Role of Nutrition in performance enhancement and postexercise recovery. J Sports Med 11: 259-267.

54. Richardson J (2004) What Patients Expect from Complementary Therapy: A Qualitative Study. Am J Public Health 94(6): 1049-1053.

55. Nagy A, McMahon A, Tapsell L, Deane F, Arenson D (2018) Therapeutic alliance in dietetic practice for weight loss: Insights from health coaching. Nutri Dietet 75(3): 250-255

56. Field A (2017) Discovering Statistics using SPSS. ( $5^{\text {th }}$ edn.), Sage Publications Ltd, London.

57. Hull SK, Page CP, Skinner BD, Linville JC, Coeytaux RR (2006) Exploring outcomes associated with acupuncture. J Altern Compl Med 12(3): $247-$ 254.
58. JMG Penedo, T Berger, M Holtforth, T Krieger, J Schröder, et al. (2020) The Working Alliance Inventory for guided Internet interventions (WAI-I). J Clin Psychol 76(6): 973-986.

59. Andrade-González, A Fernández-Liria N (2015) Spanish adaptation of the working alliance inventory (WAI). Psychometric properties of the patient and therapist forms (WAI-P and WAI-T). An Psychol 31: 524.

60. Falkenström F, Hatcher RL, Holmqvist R (2015) Confirmatory factor analysis of the patient version of the working alliance inventory-short form revised. Assess. 22(5): 581-593.

61. Pallant J (2016) SPSS Survival Manual. (6 $6^{\text {th }}$ edn.), Open University Press, New York.

62. Boeckxstaens P, Meskens A, Van der Poorten A, Verpoort AC, Sturgiss EA (2020) Exploring the Therapeutic Alliance in Belgian family medicine and its association with doctor-patient characteristics: a cross-sectional survey study. BMJ Open 10(2): e033710. 